\title{
Comprehensive Laboratory Design in the Major of Measurement and Control Technology and Instrument Facing to the Atmosphere and Ocean Field
}

\author{
Song YE ${ }^{1,}$, Long ZHANG ${ }^{2,}$ * * \\ ${ }^{1}$ College of Meteorology and Oceanography, PLA University of Science and Technology, \\ Nanjing, 211101, China \\ ${ }^{2}$ College of Meteorology and Oceanography, PLA University of Science and Technology, \\ Nanjing, 211101, China \\ aemail:yesong999@hotmail.com, bemail:fgsdhsdg@sina.com, "corresponding author
}

\begin{abstract}
Keywords: Comprehensive Laboratory, Versatility, Laboratory Design, Measurement and Control Technology and Instrument
\end{abstract}

\begin{abstract}
In this paper, the design of comprehensive laboratory related to the major of measurement and control technology and instrument in the Meteorology and Oceanography College of PLA University of science and technology was elaborated detailed. Combining with the characteristics of facing to the atmosphere and ocean field, the comprehensive laboratory design ideas and methods was proposed for saving the experiment and practice investments, improving the teaching level and qualities, cultivating cadets' practice and innovation abilities. A series of teaching and research achievements demonstrate that the comprehensive laboratory plays an important role in improving the teaching level and research ability. It can provide references for the design of comprehensive laboratory in other fields of science.
\end{abstract}

\section{Introduction}

The comprehensive laboratory was a teaching practice platform for under graduated and post graduated cadets of measurement and control technology and instrument major of PLA University of science and technology. After particularly considering our new courses: circuit design of meteorological and hydrological instruments and mechanical design of meteorological and hydrological instruments, we reconstructed our lab based on some existing experimental courses, including the principle and design of intelligent instrument, error theory and data processing, photoelectric information technology, etc., with the purpose of meeting requirements of training high level military talents.

Quantities of technical problems will be conquered, such as the accurate measurement of meteorological and hydrological factors, the control of calibrating device, the analysis of uncertainty in measurement, low power design of meteorological and hydrological instruments and the design of key components of instruments. What's clear is that the establishments and applications of some experiments, for instance, intelligent apparatus designing, measurement data processing, photoelectric measurement methods, circuit and machine design of meteorological and hydrologic instruments, will have some far-reaching impacts.

Optimizing the structure of instruments by combining the multi-type sensor, the design principle of intelligent instruments and effective methods of data processing is the important basis for accurate measurement of meteorological and hydrological instruments. The new experiment courses, such as mechanical design and drawing, automatic control, photoelectric detection, measurement and control circuit and the design of visual program, will be established. Not only can they provide the basic experimental platform for the course teaching, but also provide innovative practice platform for the application of new principle, new technology and new method in military field [1][2]. 


\section{The construction target of the laboratory}

By building comprehensive laboratory for measurement and control technology and instrument, the professional lab resources will be in full use [3]. Besides, the management of laboratory will be significantly enhanced, specialty characteristics be fully reflected in the field of meteorological and hydrological. It also can be used to provide the teaching research platform for scientific and technological innovation, which lay the foundation for talent cultivation plan.

\section{Teaching and training targets}

The laboratory can accommodate 16 cadets, which can be as an experimental teaching platform for the following courses: the principle and design of intelligent instrument, error theory and data processing, photoelectric information technology, circuit design of meteorological and hydrological instruments and mechanical design of meteorological and hydrological instruments. It also can provide innovation platform for the undergraduate cadets and graduate cadets using in innovation competitions and scientific research [4].

\section{Main functions}

Performance verification tests of intelligent instruments, including the application of I/O port, the applications of clock module, FLASH reading and writing, the application of timer, the display of $\mathrm{LED} / \mathrm{LCD}$, the operation of ADC and asynchronous communication.

Measurement experiments, including water temperature measurement, tidal measurement, humidity measurement, flow velocity measurement in wind tunnel, combined measurement of meteorological factor, principle analysis of laser range finder, bubble measurement experiment in water tank, the experiment of data processing and error analysis, area measurement of two dimensional complex object using CCD.

The design of comprehensive experiments, including manual drawing, design and machining of instrument shell, design and implementation of infrared and visible light emitting circuit, design and implementation of infrared receiving circuit and response measurement, design and implementation of the detection system for light emitting diode and laser, low power design of meteorological and hydrological instruments, design and processing of complex structure model, design and forming of precision parts, 3D design and molding by computer.

This lab has the carrying capacity to load five experimental equipment, provide basic conditions for laboratory teaching and scientific research.

\section{The construction target of the laboratory}

\section{Laboratory design of spatial flexibility}

The combination of teaching function and laboratory space can deepen the teaching; promote the communication between teachers and cadets [5]. Flexible space design made the laboratory adapted to different functions and practical requirements. The wide communication space can stimulate the interaction between teachers and cadets. In addition, the laboratory can also accommodate all the experimental instruments and equipment by configuring the storage space reasonably.

According to different teaching content, the space layout of the comprehensive laboratory can be divided into three kinds as shown in Figure 1 to Figure 3. The layout of the ground power and wall power is shown in Figure 4. The functional areas of this laboratory are shown in Figure 5. It distinctly reflects the design idea of reconfigurable and multi-functional.

\section{The construction of experimental platform based on five professional course}

According to the actual function in the experimental platform, the experimental equipment can be divided into six categories: measurement standard, measurement class, control class, machinery class, comprehensive class and components of meteorological and hydrological instruments. The structures of all kinds of experimental instruments are shown in Figure 6. The scientific classification of the experimental instruments is not only beneficial to the storage, use and management of the equipment, but also save the investment [6]. 


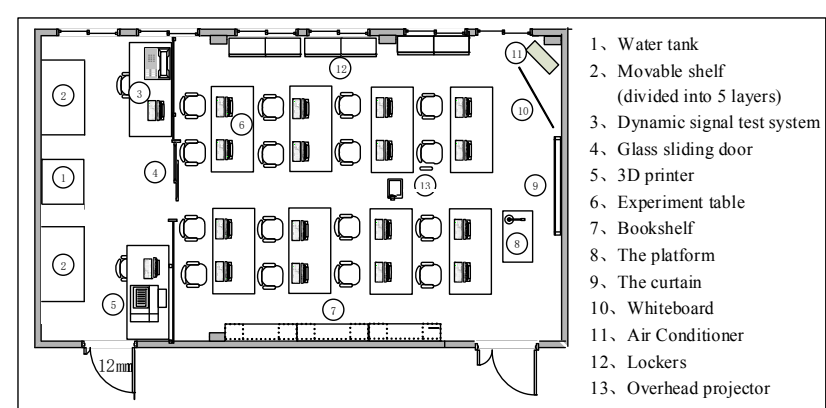

Fig. 1. The space layout of data processing and instrument circuit.

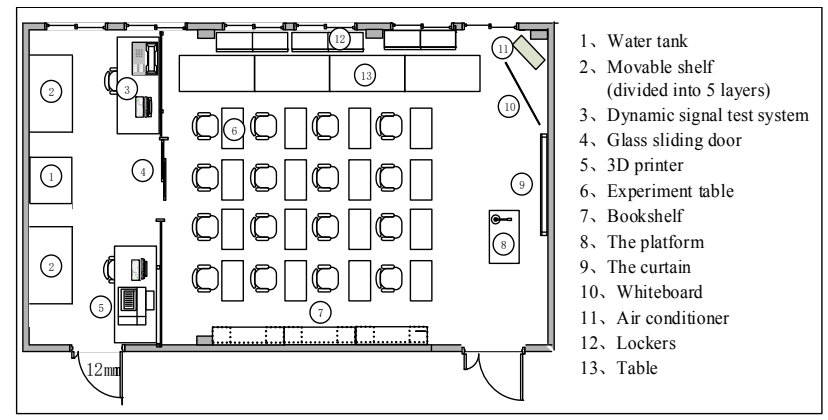

Fig. 3. The space layout of manual mapping experiment.

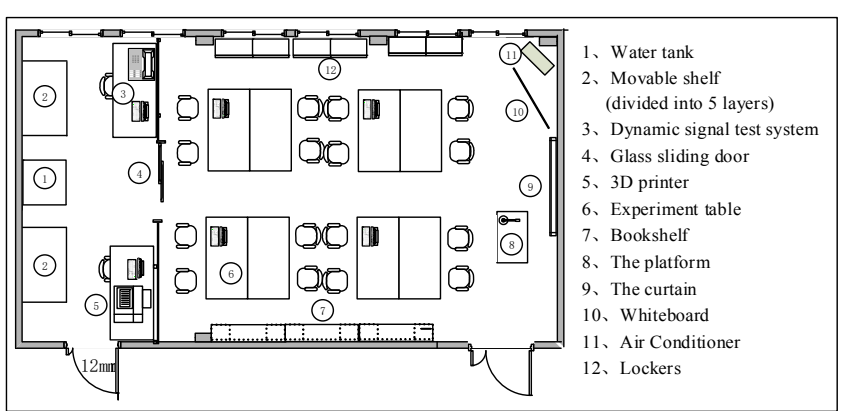

Fig. 2. The space layout of photoelectric experiment and mechanical.

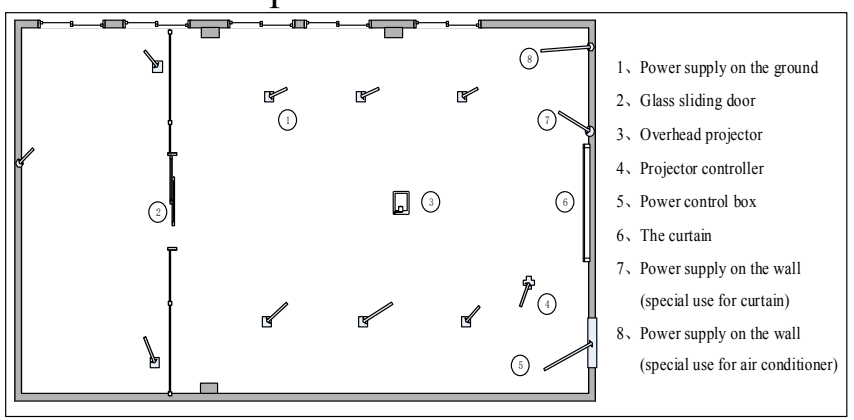

Fig. 4. The space layout of power.

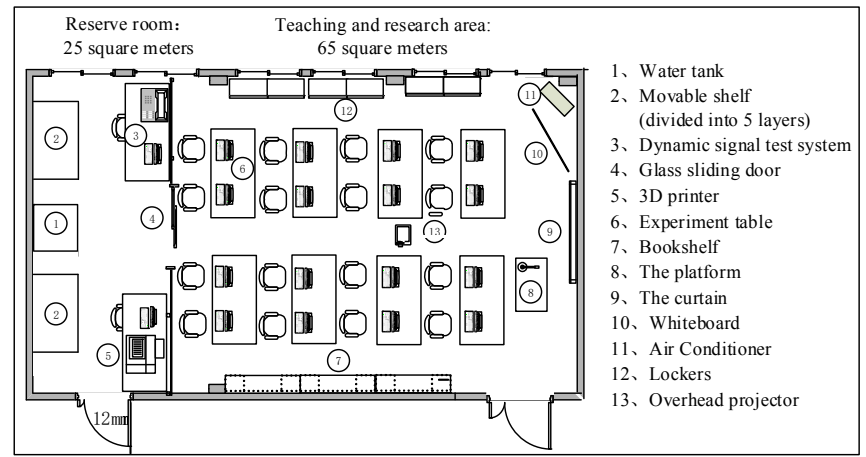

Fig. 5. Functional area division of laboratory.
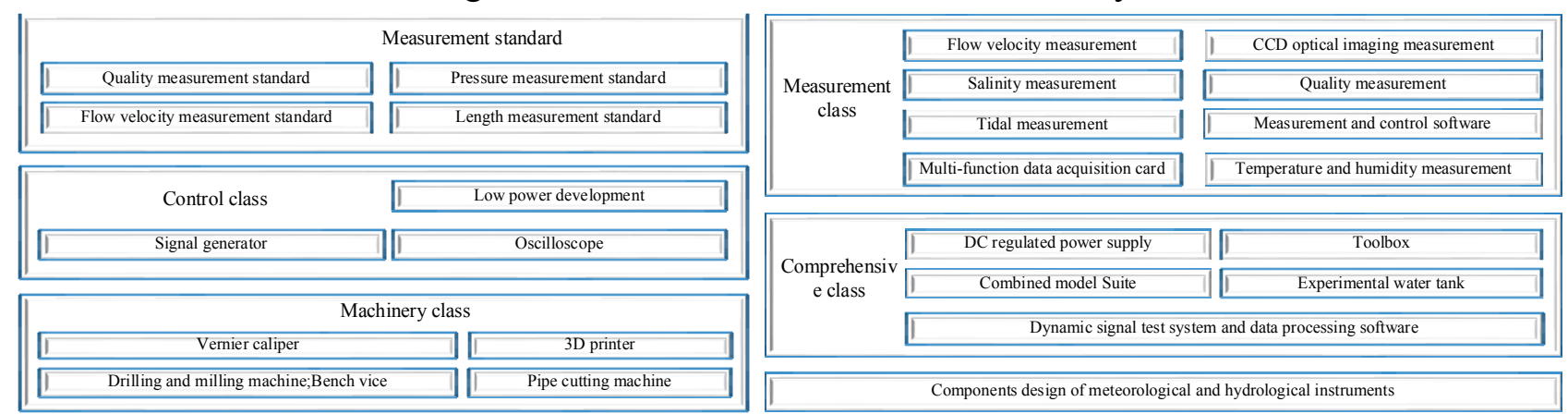

Fig. 6. Classification of instruments in the comprehensive laboratory.

The experimental platform for intelligent instrument principle and design. This experimental platform mainly consists of the measurement, control, comprehensive class and components of meteorological and hydrological instruments. The five important experiments are shown in Figure 7. This course has obvious characteristics of comprehensive and practical with the purpose of strengthening the ability of development and design for an instrument system using the relevant knowledge and technology, and cultivating cadets' ability to develop products and do research independently. The requirement of this experiment is to design the hardware and software system according to the instrument design methods, as well as debug the system using development tools.

The experimental platform for error theory and data processing. The experimental platform of this course mainly consists of the measurement class, measurement standard and the 
comprehensive class. The five experiments shown in Figure 8 are the key points in teaching. According to the requirement of measurement accuracy, cadets can throw themselves into the open experiment, and finish the data processing and analysis with the assistance of computer program.

The experimental platform for photoelectric information technology. This experimental platform includes the measurement and comprehensive experimental equipment and components in meteorological and hydrological instruments. The five experiments shown in Figure 9 are the key points in teaching. Cadets can choose to do any one of the topics according to their own interests, reflecting the concept of personalized education. As a curriculum design, the experiment will be included in the category of autonomous learning.

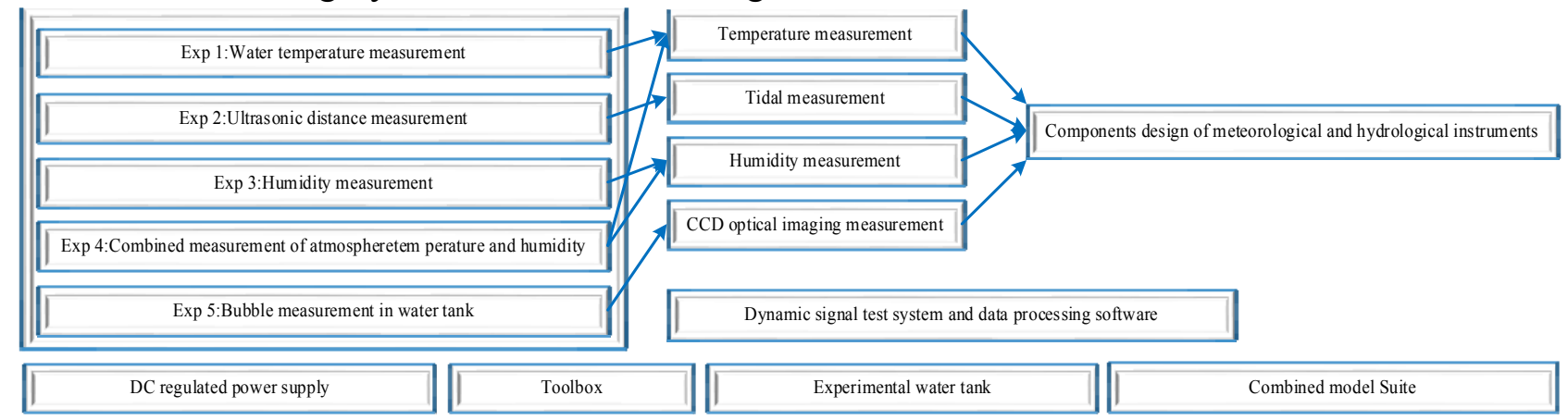

Fig. 7. The relationship diagram of instruments in the experiment of "intelligent instrument principle and design".

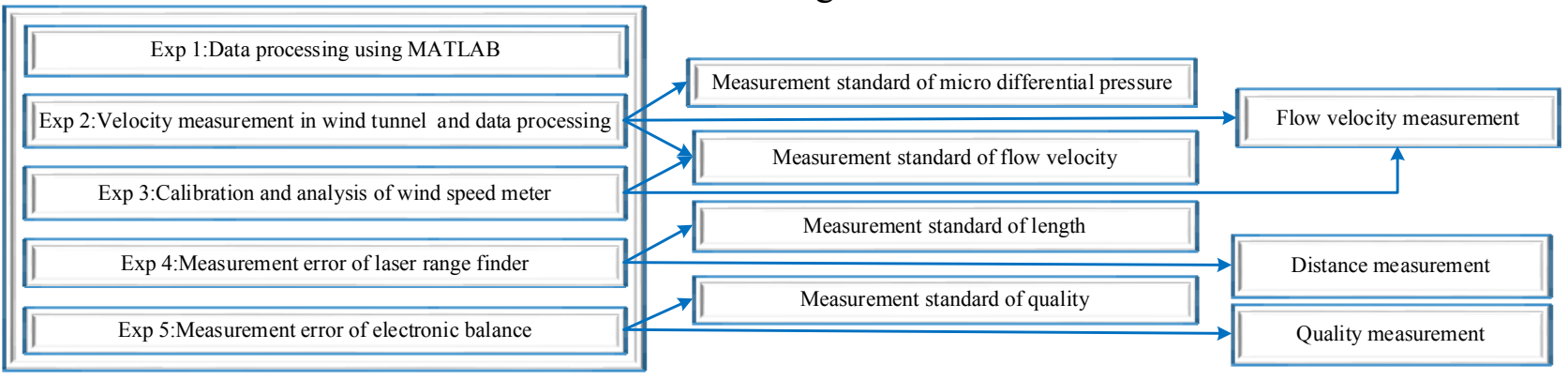

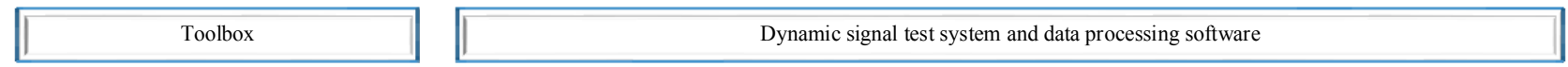

Fig. 8. The relationship diagram of instruments in the experiment of "error theory and data processing".

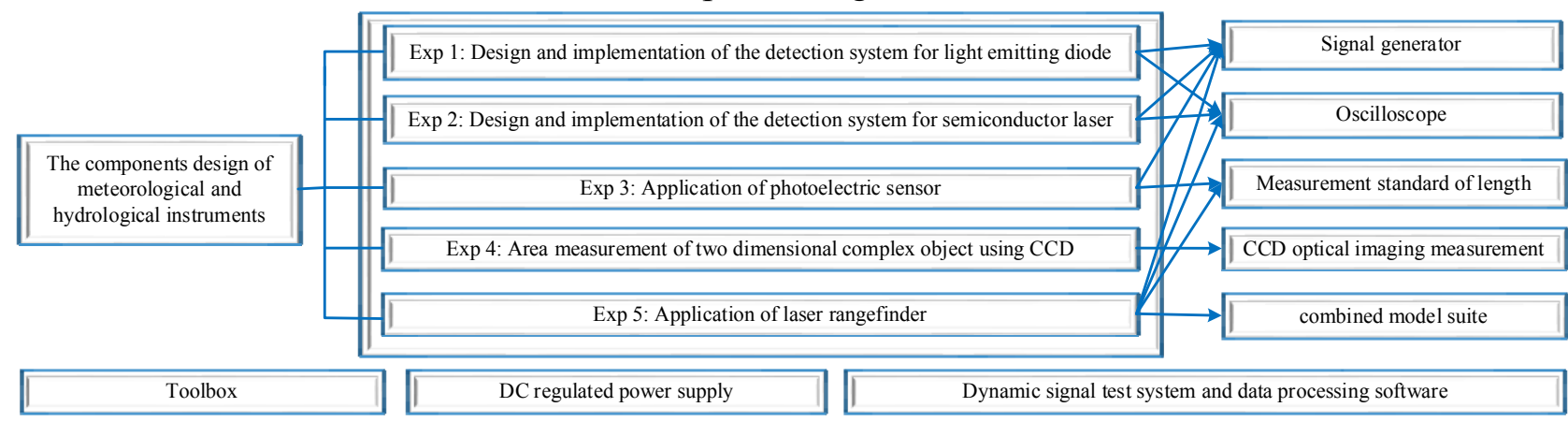

Fig. 9. The relationship diagram of instruments in the experiment of "photoelectric information technology".

The experimental platform for the circuit design of the meteorological and hydrological instruments. This experimental platform includes the control and comprehensive experimental equipment and components in meteorological and hydrological instruments. The seven experiments shown in Figure 10 are the key points in teaching. This experiment is divided into basic experiment, improving experiment and research design experiment with the purpose of cultivating the cadets' abilities of analyzing and solving problems. The basic experiments include FLASH read and write, LED/LCD display and the applications of I/O port, clock module and timer. The improving experiments include ADC operation experiment and asynchronous communication experiment; the 
design and research experiment is the low power design of meteorological and hydrological instruments.

The experimental platform for the mechanical design of meteorological and hydrological instruments. This experimental platform includes the control, mechanics and comprehensive experimental equipment. The five experiments shown in Figure 11 are the key points in teaching. The combination of drawing and material objects can cultivate cadets' abilities of spatial imagination, help them understand the design ideas for common meteorological and hydrological instruments, and encourage cadets to carry out innovation experiments independently.

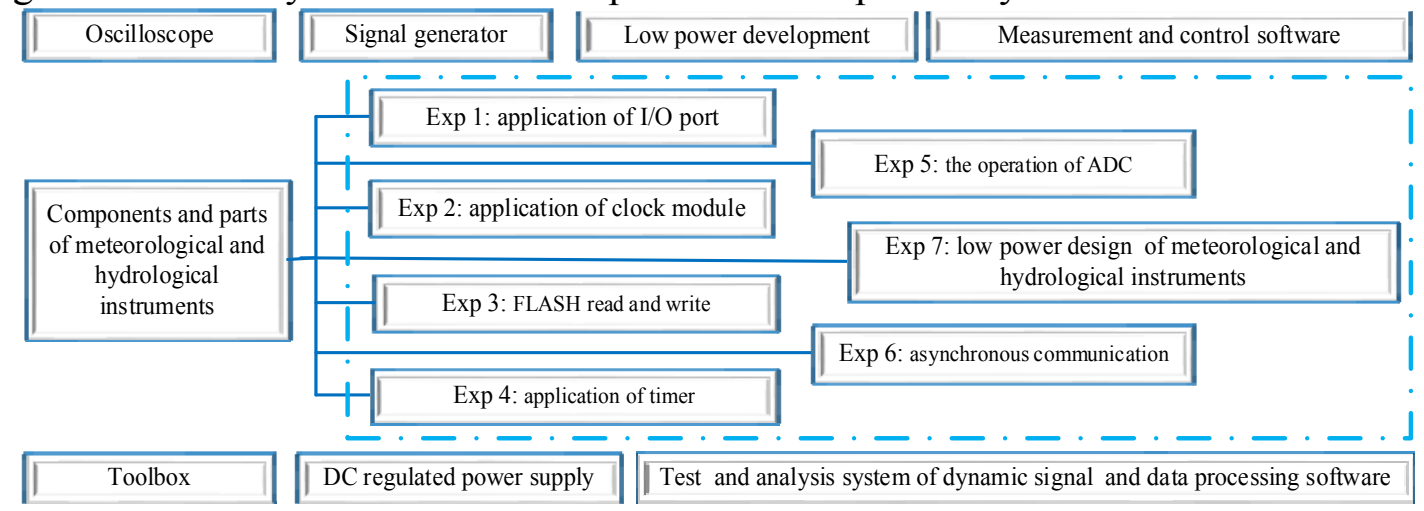

Fig. 10. The relationship diagram of instruments in the experiment of "the circuit design of the meteorological and hydrological instruments".

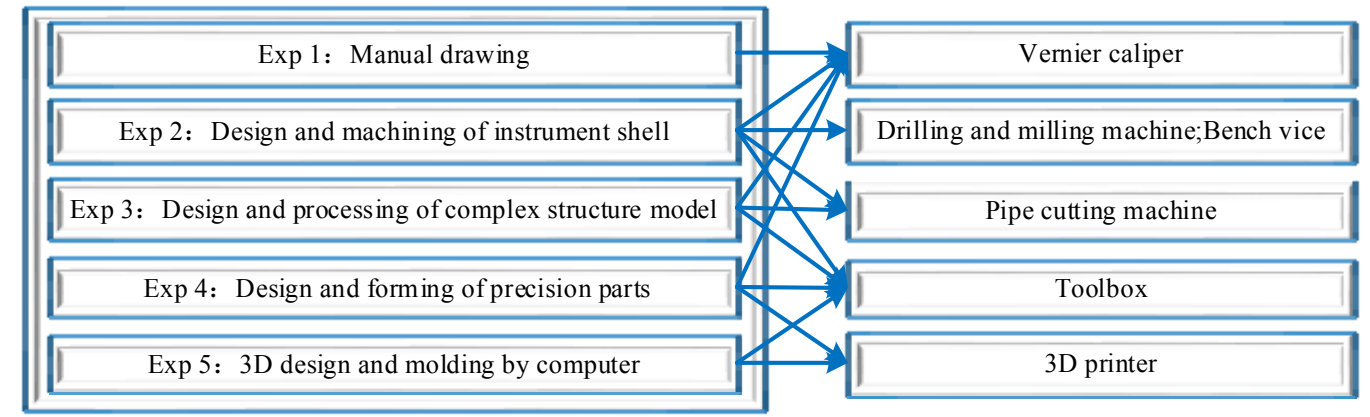

Fig. 11. The relationship diagram of instruments in the experiment of "the mechanical design of the meteorological and hydrological instruments".

Comprehensive innovation experiment platform. In the process of experiments, the cadets combine theory knowledge with the practice, and develop their innovative ideas and methods. The design results of meteorological and hydrological instruments can be analyzed using the dynamic signal testing and analysis system. And the mechanical design results can be tested using 3D printer. With the promotion of innovation competition, the laboratory will create more innovative results and open up a new path for the cultivation of innovative talents.

The application of dynamic signal test system and 3D printer can cultivate cadets' comprehensive ability and innovation consciousness [7]. In the process of experiments, cadets will have a better understanding for the mechanical processing, electronic circuits, software programming and other related knowledge. The data obtained from these experiments can be used as the original data in the experiment of error theory and data processing.

The laboratory created a broad platform for the cadets on mechanical transmission, programming control, CAD/CAM computer aided design, electronic technology and other related courses. The operating mode of the laboratory meets the concept of teaching methods innovation [8].

\section{Conclusion}

The construction of comprehensive laboratory for the major of measurement and control technology and instrument is a complicated systematic engineering, which involves the major development planning, organic integration of theory and experiment teaching, laboratory management and the improvement of equipment utilization. In addition, the joint efforts of teachers, cadets, as well as 
management departments are also needed. There are many problems needed to further studying and deeply exploring. For example, the laboratory's characteristics for the field of atmosphere and ocean, designing of multi-function experiment platform and forming a complete experimental teaching model.

\section{Acknowledgment}

The work in this paper could not have been presented without the efforts of numerous people. The author would like to thank the Education and Training Program of PLA University of Science and Technology. LIU Feng and LI Jun are acknowledged for stimulating discussions. The guidance from Professor WANG Xiao-Lei and Associate professor YANG Chang-Ye, together with the constructive comments from CHEN Zhen-Tao and CHEN Xiao-Ying, are gratefully acknowledged.

\section{References}

[1] Pan Xin-ji. Research and Application of Laboratory Open Management System of Universities. Research and Exploration in Laboratory. 2009, 28(9): pp.143-156.

[2] Han Chun-tian. Discussion on laboratory construction and management work in colleges and universities. Experimental Technology and Management. 2011, 28(8): pp.189-191.

[3] Jail Hal-li, Song Duan-zhi, Feng Xuan-jun. The Application of Web-Based Virtual Reality Labs. Computer engineering and science. 2002, 24(2): pp.46-48.

[4] Jiang Jing-Hua. Discussion on improvement of Lab's Investment Efficiency. Research and Exploration in Laboratory. 2004, 23(5): pp. 1-5.

[5] He Jin-zhe, Xu Jing-boo. Study and exploration on resource sharing mechanism of laboratory. Laboratory Science. 2010, 6(13): pp.132-133.

[6] Liu Hui. Shallow theory colleges equipment resource sharing. China Modern Educational Equipment. 2006, 8: pp.67-69.

[7] Liu Xiang-yang, Zheng Hai-woo. Research about Construction and Reform of Specialized Laboratory. Research and Exploration in Laboratory. 2010, 29(2): pp.145-148.

[8] Wei Dong-may, etc. Strengthening laboratory management to improve the quality of experimental teaching. Colleges Laboratory Research. 2010, 3: pp.31. 